\title{
Cumulative thermonuclear inertial reactor
}

\author{
Alexander Bolonkin
}

\begin{abstract}
Background: In the last 60 years, the scientists spent tens of billion dollars attempting to develop useful thermonuclear energy. But they cannot yet reach a stable thermonuclear reaction. They still are promising publicly, after another 15-20 years, more tens of billions of US dollars to finally design the expensive workable industrial installation.

The author offers a new, small, cheap cumulative inertial thermonuclear reactor, which increases the pressure and temperature of its nuclear fuel thousands of times, reaches the required ignition stage and, ultimately, full constant contained thermonuclear reaction. Cumulative reactor contains several innovations to achieve its product. It is safe and ecological. The author is aware that no cumulative pressure research was done anywhere else in the world, especially with additional rocket acceleration.
\end{abstract}

Methods: The chief among them is using moving explosives (rocket thrust), which allows to accelerate the special piston to a very high speed (more $30 \mathrm{~km} / \mathrm{s}$ ) which (as it is shown by integral computations) compresses the fuel capsule a million times and heats up million degrees of temperature.

Results: The author gives detailed computations of the Lawson criterion. He shows the current conventional inertial laser method gives only $0.28<1$ of the needed Lawson criterion of thermonuclear reaction $\left(\rho R>1 \mathrm{~g} / \mathrm{cm}^{2}\right)$. The offered method gives $585>1$ of the needed Lawson criterion.

Conclusions: The proposed method cannot only achieve but also significantly exceed the Lawson criterion. The experimental reactor is about a thousand times cheaper than a typical laser unit.

Keywords: Micro-thermonuclear reactor, Cumulative AB thermonuclear reactor, Transportation thermonuclear reactor, Aerospace thermonuclear engine, Nuclear rockets, Nuclei fuse

\section{Background}

\section{Brief information about thermonuclear reactors}

Fusion power is useful energy generated by nuclear fusion reactions. In this kind of reaction, two light atomic nuclei fuse together to form a heavier nucleus and release energy. The largest current nuclear fusion experiment, JET, has resulted in fusion power production somewhat larger than the power put into the plasma, maintained for a few seconds. In June 2005, the construction of the experimental reactor International Thermonuclear Experimental Reactor (ITER), designed to produce several times more fusion power than the power into it generating the plasma over many minutes, was announced. The unrealized production of net electrical power from fusion machines is planned for the next-generation experiment after ITER.

Correspondence: abolonkin@gmail.com

Consultation and Research Co., City College of New York, New York, NY, USA
Unfortunately, this task is not easy, as scientists thought early on. Fusion reactions require a very large amount of energy to initiate in order to overcome the so-called Coulomb barrier or fusion barrier energy. The key to practical fusion power is to select a fuel that requires the minimum amount of energy to start, that is, the lowest barrier energy. The best fuel from this standpoint is a one-to-one mix of deuterium and tritium; both are heavy isotopes of hydrogen. The D-T (deuterium and tritium) mix has suitable low barrier energy. In order to create the required conditions, the fuel must be heated to tens of millions of degrees and/or compressed to immense pressures.

At present, D-T is used by two main methods of fusion: inertial confinement fusion (ICF) and magnetic confinement fusion (MCF) - for example, tokomak device.

Inertial confinement fusion (ICF). In ICF, nuclear fusion reactions are initiated by heating and compressing a target. The target is a pellet that most often contains deuterium and tritium (often only micro- or milligrams). 
Intense focused laser or ion beams are used for compression of pellets. The beams explosively detonate the outer material layers of the target pellet. That accelerates the underlying target layers inward, sending a shock wave into the center of each pellet's mass. If the shock wave is powerful enough, and if high enough density at the center is achieved, some of the fuel will be heated enough to cause pellet fusion reactions. In a target which has been heated and compressed to the point of thermonuclear ignition, energy can then heat surrounding fuel to cause it to fuse as well, potentially releasing tremendous amounts of energy.

Magnetic confinement fusion (MCF). Since plasmas are very good electrical conductors, magnetic fields can also be configured to safely confine fusion fuel. A variety of magnetic configurations can be used, the basic distinction being between magnetic mirror confinement and toroidal confinement, especially tokomaks and stellarators.

Lawson criterion. In nuclear fusion research, the Lawson criterion, first derived by John D. Lawson in 1957, is an important general measure of a system that defines the conditions needed for a fusion reactor to reach ignition stage, that is, the heating of the plasma by the products of the fusion reactions is sufficient to maintain the temperature of the plasma against all losses without external power input. As originally formulated, the Lawson criterion gives a minimum required value for the product of the plasma (electron) density $n_{\mathrm{e}}$ and the "energy confinement time" $\tau$. Later analyses suggested that a more useful figure of merit is the "triple product" of density, confinement time, and plasma temperature $T$. The triple product also has a minimum required value, and the name "Lawson criterion" often refers to this important inequality.

The key to practical fusion power is to select a fuel that requires the minimum amount of energy to start, that is, the lowest barrier energy. The best known fuel from this standpoint is a one-to-one mix of deuterium and tritium; both are heavy isotopes of hydrogen. The D-T mix has a low barrier.

In order to create the required conditions, the fuel must be heated to tens of millions of degrees, and/or compressed to immense pressures. The temperature and pressure required for any particular fuel to fuse is known as the Lawson criterion. For the D-T reaction, the physical value is about

$$
\begin{aligned}
& L=n_{\mathrm{e}} T \tau>\left(10^{14} \div 10^{15}\right) \text { in "cgs" units } \\
& \text { or } L=n T \tau>\left(10^{20} \div 10^{21}\right) \text { in CI units }
\end{aligned}
$$

where $T$ is the temperature, $[\mathrm{KeV}], 1 \mathrm{eV}=1.16 \times 10^{4} \mathrm{~K}$; $n_{\mathrm{e}}$ is the matter density, $\left[1 / \mathrm{cm}^{3}\right] ; n$ is the matter density, $\left[1 / \mathrm{m}^{3}\right]$; and $\tau$ is the time, $[\mathrm{s}]$. The last equation is in metric system. The thermonuclear reaction of ${ }^{2} \mathrm{H}+{ }^{3} \mathrm{D}$ realizes if $L>10^{20}$ in CI (meter, kilogram, second) units or $L>10^{14}$ in "cgs" (centimeter, gram, second) units.
This number has not yet been achieved in any fusion reactor, although the latest generations of fusion-making machines have come significantly close to doing so. For instance, the reactor TFTR has achieved the densities and energy lifetimes needed to achieve Lawson at the temperatures it can create, but it cannot create those temperatures at the same time. Future ITER aims to do both.

The Lawson criterion applies to inertial confinement fusion as well as to magnetic confinement fusion but is more usefully expressed in a different form. Whereas the energy confinement time in a magnetic system is very difficult to predict or even to establish empirically, in an inertial system, it must be on the order of the time it takes sound waves to travel across the plasma:

$$
\tau \approx \frac{R}{\sqrt{k T / m_{i}}}
$$

where $\tau$ is the time, $\mathrm{s} ; R$ is the distance, $\mathrm{m} ; k$ is the Boltzmann constant; $T$ is the temperature, $\mathrm{K}$; and $m_{\mathrm{i}}$ is the mass of ion, $\mathrm{kg}$.

Following the above derivation of the limit on $n_{\mathrm{e}} \tau_{\mathrm{E}}$, we see that the product of the density and the radius must be greater than a value related to the minimum of $T^{3 / 2} /$ $<\sigma v\rangle$ (here, $\sigma$ is the Boltzmann constant, $v$ is the ion speed). This condition is traditionally expressed in terms of the mass density $\rho$ :

$$
\rho R>1 \mathrm{~g} / \mathrm{cm}^{2}
$$

To satisfy this criterion at the density of solid, D + T $\left(0.2 \mathrm{~g} / \mathrm{cm}^{3}\right)$ would require implausibly large laser pulse energy. Assuming the energy required scales with the mass of the fusion plasma $\left(E_{\mathrm{laser}} \sim \rho R^{3} \sim \rho^{-2}\right)$, compressing the fuel to $10^{3}$ or $10^{4}$ times solid density would reduce the energy required by a factor of $10^{6}$ or $10^{8}$, bringing it into a realistic range. With a compression by $10^{3}$, the compressed density will be $200 \mathrm{~g} / \mathrm{cm}^{3}$, and the compressed radius can be as small as $0.05 \mathrm{~mm}$. The radius of the fuel before compression would be $0.5 \mathrm{~mm}$. The initial pellet will be perhaps twice as large since most of the mass will be ablated during the compression stage by a symmetrical energy input bath.

The fusion power density is a good figure of merit to determine the optimum temperature for magnetic confinement, but for inertial confinement, the fractional burn-up of the fuel is probably more useful. The burn-up should be proportional to the specific reaction rate $\left(n^{2}<\sigma v>\right)$ times the confinement time (which scales as $T^{1 / 2}$ ) divided by the particle density $n$ : burn-up fraction $\sim n^{2}<\sigma \mathrm{V}>T^{-1 / 2} / n \sim(n T)\left(<\sigma \mathrm{V}>/ T^{3 / 2}\right)$.

Thus, the optimum temperature for inertial confinement fusion is that which maximizes $<\sigma v>/ T^{3 / 2}$, which is slightly higher than the optimum temperature for magnetic confinement. 
Short history of thermonuclear fusion. One of the earliest (in the late 1970s and early 1980s) serious attempts at an ICF design was Shiva, a 20-armed neodymium laser system built at the Lawrence Livermore National Laboratory (LLNL) in California that started operation in 1978. Shiva was a "proof of concept" design, followed by the NOVA design with 10 times the power. Funding for fusion research was severely constrained in the 1980s, but NOVA nevertheless successfully gathered enough information for a next-generation machine whose goal was ignition. Although net energy can be released even without ignition (the breakeven point), ignition is considered necessary for a practical power system.

The resulting design, now known as the National Ignition Facility, commenced being constructed at LLNL in 1997. Originally intended to start construction in the early 1990s, the National Ignition Facility (NIF) is now 6 years behind schedule and over-budget by some $\$ 3.5$ billion. Nevertheless, many of the problems appear to be due to the "Big Science Laboratory" mentality, shifting the focus from pure ICF research to the nuclear stewardship program, LLNLs traditional nuclear weapons-making role. NIF "burned" in 2010, when the remaining lasers in the 192-beam array were finally installed. Like those earlier experiments, however, NIF has failed to reach ignition and is, as of 2015, generating only about one third of the required energy levels needed to reach the full fusion stage of the operation.

Laser physicists in Europe have put forward plans to build a $£ 500 \mathrm{~m}$ facility, called HiPER, to study a new approach to laser fusion. A panel of scientists from seven European Union countries believes that a "fast ignition" laser facility could make a significant contribution to fusion research, as well as supporting experiments in other areas of physics.
The facility would be designed to achieve high-energy gains, providing the critical intermediate step between ignition and a demonstration reactor. It would consist of a longpulse laser with energy of $200 \mathrm{~kJ}$ to compress the fuel and a short-pulse laser with energy of $70 \mathrm{~kJ}$ to heat it.

Confinement refers to all the conditions necessary to keep plasma dense and hot long enough to undergo fusion:

- Equilibrium: There must be no net forces on any part of the plasma; otherwise, it will rapidly disassemble. The exception, of course, is inertial confinement, where the relevant physics must occur faster than the disassembly time.

- Stability: The plasma must be so constructed that small deviations are restored to the initial state; otherwise, some unavoidable disturbance will occur and grow exponentially until the plasma is destroyed.

- Transport: The loss of particles and heat in all channels must be sufficiently slow. The word "confinement" is often used in the restricted sense of "energy confinement".

To produce self-sustaining fusion, the energy released by the reaction (or at least a fraction of it) must be used to heat new reactant nuclei and keep them hot long enough that they also undergo fusion reactions. Retaining the heat generated is called energy confinement and may be accomplished in a number of ways.

Hydrogen bomb weapons require no confinement at all. The fuel is simply allowed to fly apart, but it takes a certain length of time to do this, and during this time, fusion can occur. This approach is called inertial confinement (Fig. 1). If more than about a milligram of fuel

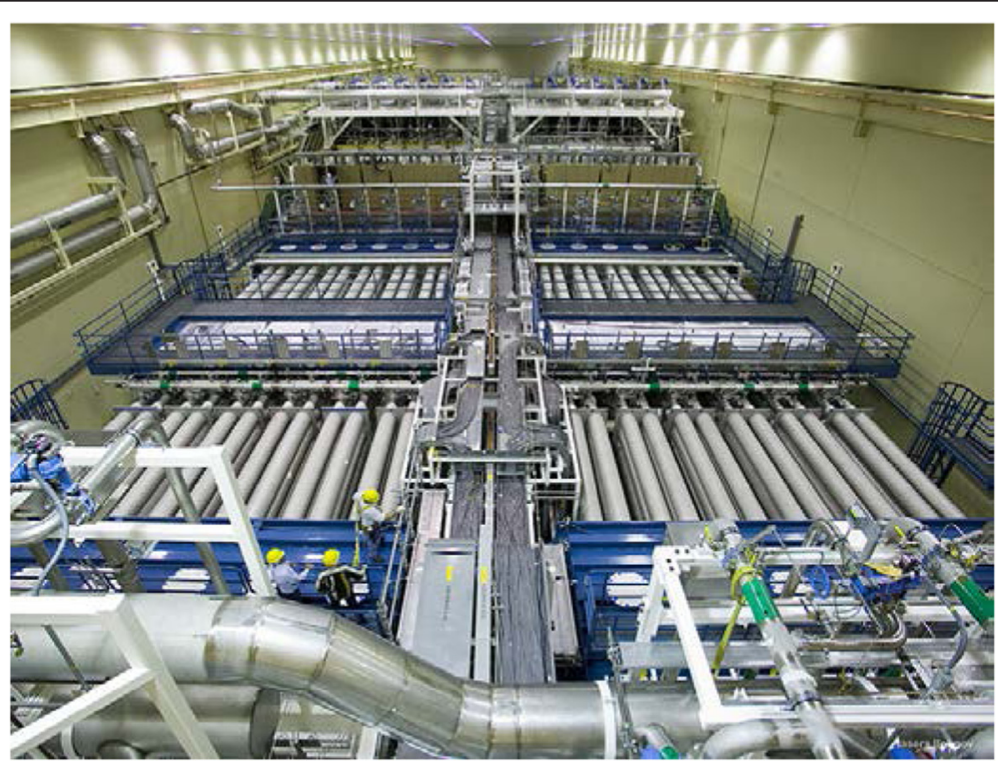

Fig. 1 One laser installation of NIF. You can see people in the installation 
is used, the explosion would destroy the machine, so controlled thermonuclear fusion using inertial confinement causes tiny pellets of fuel to explode several times a second. To induce the explosion, the pellet must be compressed to about 30 times solid density with energetic beams. If the beams are focused directly on the pellet, it is called direct drive, which can in principle be very efficient, but in practice, it is difficult to obtain the needed uniformity. An alternative approach is indirect drive, in which the beams heat a shell, and the shell radiates $\mathrm{x}$-rays, which then implode the pellet. The beams are commonly laser beams, but heavy and light ion beams and electron beams have all been investigated and tried to one degree or another.

They rely on fuel pellets with a "perfect" globular shape in order to generate a symmetrical inward shock wave to produce the high-density plasma, and in practice these have proven difficult to produce. A recent development in the field of laser-induced ICF is the use of ultra-shortpulse multi-petawatt lasers to heat the plasma of an imploding pellet at exactly the moment of greatest density after it is imploded conventionally using terawatt-scale lasers. This research will be carried out on the (currently being built) OMEGA EP petawatt and OMEGA lasers at the University of Rochester in New York and at the GEKKO XII laser at the Institute for Laser Engineering in Osaka, Japan, which, if fruitful, may have the effect of greatly reducing the cost of a laser fusion-based power source.

At the temperatures required for fusion, the fuel is in the form of plasma with very good electrical conductivity. This opens the possibility to confine the fuel and the energy with magnetic fields, an idea known as magnetic confinement (Fig. 2).

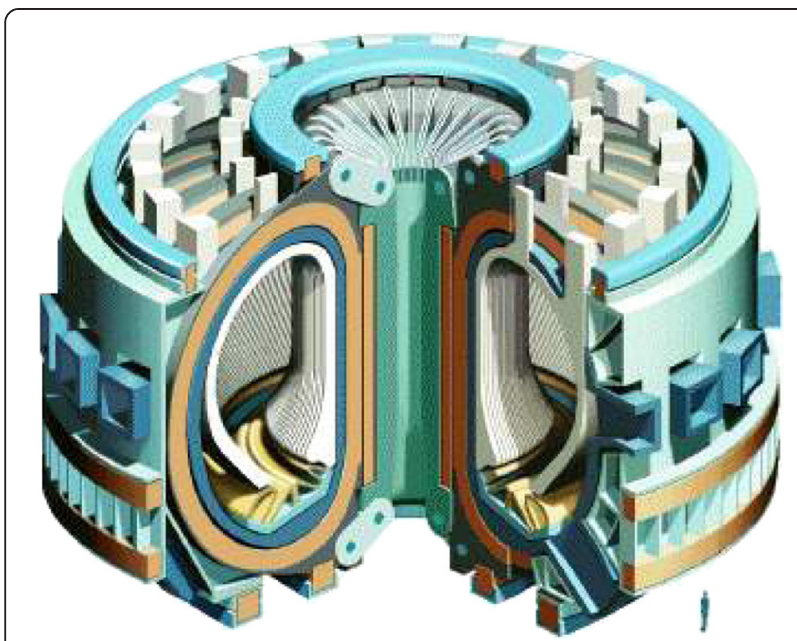

Fig. 2 Magnetic thermonuclear reactor. The size of the installation is obvious if you compare it with the "Little Blue Man" inside the machine at the bottom. Cost is some tens of billions of dollars
Much of this progress has been achieved with a particular emphasis on tokomaks (Fig. 2).

In fusion research, achieving a fusion energy gain factor $Q=1$ is called breakeven and is considered a significant although somewhat artificial milestone. Ignition refers to an infinite $Q$, that is, a self-sustaining plasma where the losses are made up for by fusion power without any external input. In a practical fusion reactor, some external power will always be required for things like current drive, refueling, profile control, and burn control. A value on the order of $Q=20$ will be required if the plant is to deliver much more energy than it uses internally.

In a fusion power plant, the nuclear island has a plasma chamber with an associated vacuum system, surrounded by a plasma-facing components (first wall and diverter) maintaining the vacuum boundary and absorbing the thermal radiation coming from the plasma, surrounded in turn by a blanket where the neutrons are absorbed to breed tritium and heat a working fluid that transfers the power to the balance of plant. If magnetic confinement is used, a magnet system, using primarily cryogenic superconducting magnets, is needed, and usually systems for heating and refueling the plasma and for driving current. In inertial confinement, a driver (laser or accelerator) and a focusing system are needed, as well as a means for forming and positioning the pellets.

The magnetic fusion energy (MFE) program seeks to establish the conditions to sustain a nuclear fusion reaction in plasma that is contained by magnetic fields to allow the successful production of fusion power.

In 30 years, scientists have increased the Lawson criterion of the ICF and tokomak installations by tens of times. Unfortunately, all current and some new installations (ICF and tokomak) have a Lawrence criterion that is tens of times lower than is necessary (Fig. 3).

Data of same current inertial laser installations:

1. NOVA uses laser NIF (USA), has 192 beams, impulse energy up $120 \mathrm{~kJ}$. It reaches a density of $20 \mathrm{~g} / \mathrm{cm}^{3}$, and its speed of cover is up $300 \mathrm{~km} / \mathrm{s}$. NIF has failed to reach ignition and is, as of 2013 , generating about one third of the required energy levels. NIF cost is about \$3.5B.

2. YiPER (EU) has impulse energy up $70 \mathrm{~kJ}$.

3. OMEGA (USA) has impulse energy up $60 \mathrm{~kJ}$.

4. GEKKO-XII (Japan) has impulse energy up $20 \mathrm{~kJ}$. It reaches a density of $120 \mathrm{~g} / \mathrm{cm}^{3}$.

5. Febus (France) has impulse energy up $20 \mathrm{~kJ}$.

6. Iskra-5 (Russia) has impulse energy up $30 \mathrm{~kJ}$.

The proposed reactor is safe because it uses milligrams of a nuclear fuel. A thick sphere protects the environment from possible few radiation and 


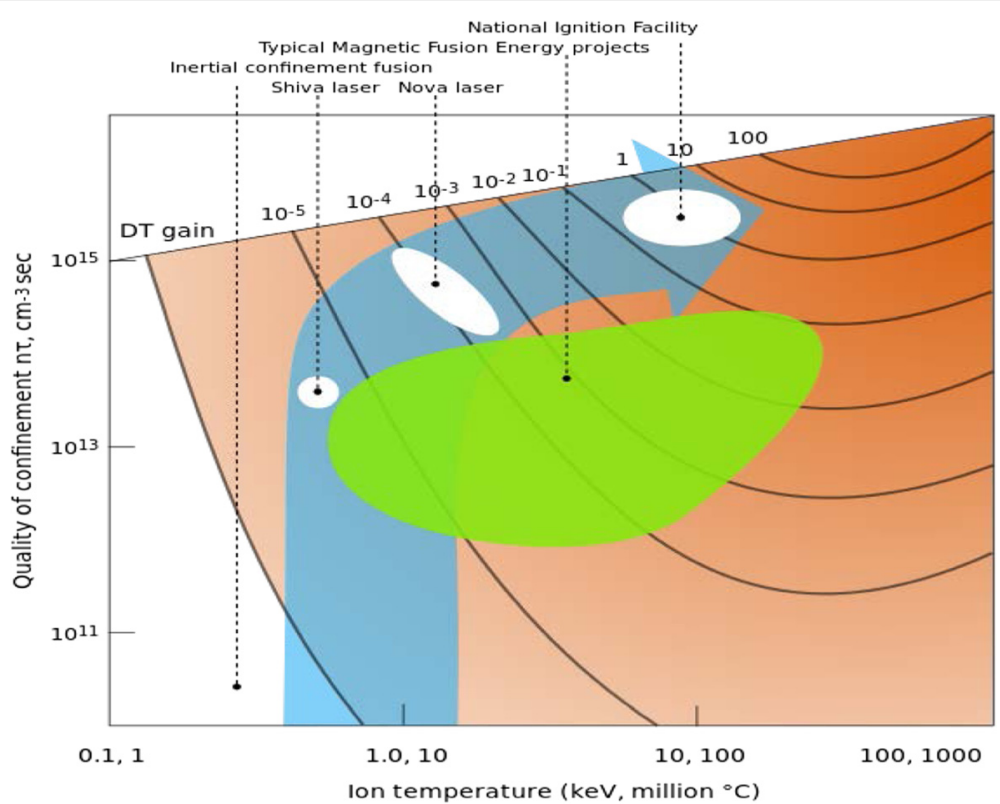

Fig. 3 Parameter space occupied by inertial fusion energy and magnetic fusion energy devices. The regime allowing thermonuclear ignition with high gain lies near the upper right corner of the plot

radioactive fallout. The reactor is small and may be used in sea ships, submarines, aircrafts, rockets, and trains.

\section{Methods}

\section{Description and innovations of cumulative $A B$ reactors Description}

Laser method disadvantages Thermonuclear reactors and, in particular, laser methods have been under development for about 60 years. Governments have already spent tens of billions of US dollars, but it is not yet seen as an industrial application of thermonuclear energy for the coming 10-15 years time period. The laser has very low efficiency (1-1.5 \%), acts at a very shot time $\left(10^{-9}-10^{-10} \mathrm{~s}\right)$, and has enough energy that is not delivered to the center of the spherical fuel pellet, and there are a lot of future problems in the radioactivity and in converting the thermonuclear energy into useful energy.

Cumulative method The author offers the new method, which is cheaper by thousands of times, is more efficiency, and does not have many disadvantages of the laser and magnetic methods. Detailed consideration of the advantages of the new method and computation proofs will be in next the paragraph.

Description of new reactor and method The most comfortable version 1 of the cumulative $A B$ thermonuclear reactor is presented in Figs. 4, 5, and 6.
The new thermonuclear reactor contains the following(Fig. 4): strong sphere 1, fuse 2 , six (or more) explosive injectors 3 , piston 4 from heavy appropriate material, fuel pellet 5, and tube 6 for loading and primary acceleration.

The cumulative $\mathrm{AB}$ reactor works in the following way (Figs. 5, 6, and 7). The net fuses 2 (six or more) (Fig. 4)

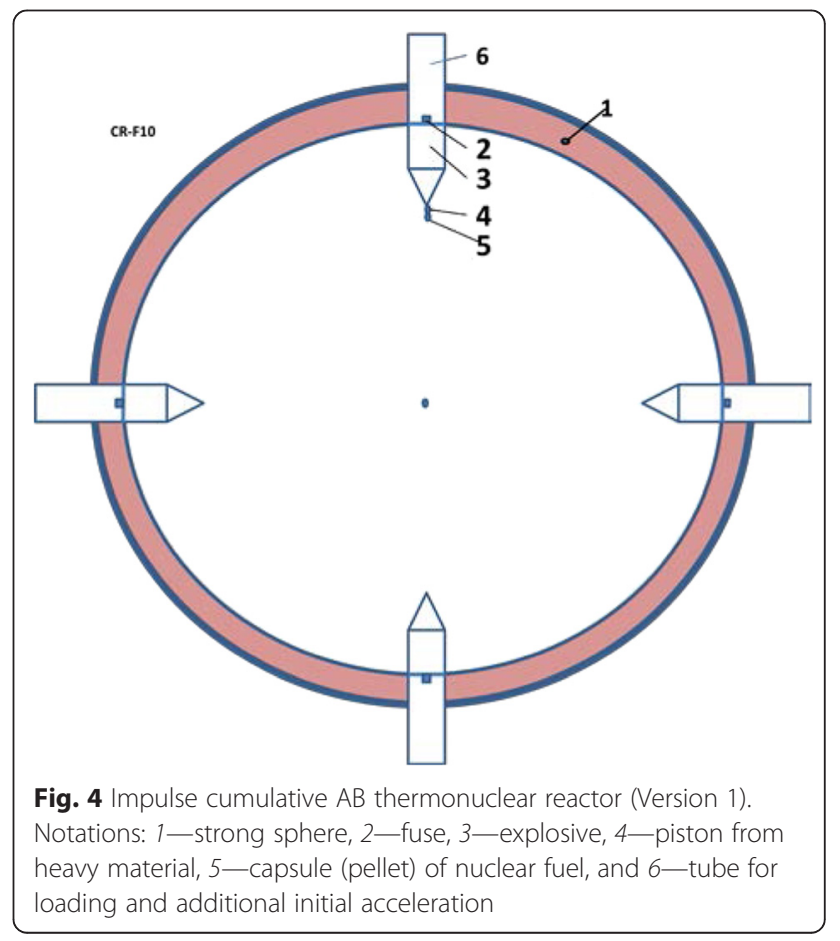



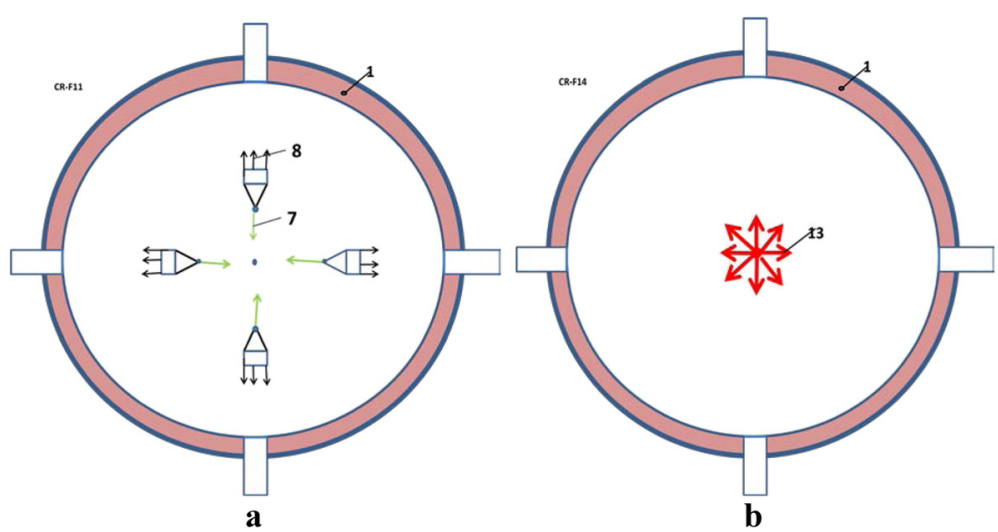

Fig. 5 Work of $A B$ thermonuclear reactor (Version 1). Notations: (a) rocket acceleration of pistons, (b) nuclear explosion; 7 is moving of explosive and pistons (force from rocket thrust), 8 is flow of rocket explosive gas, and 13-thermonuclear explosion

simultaneously blows the nearest layer of the explosive 3 (shoot, fire off), and an explosive gas is pushing the explosive 3 to center of sphere (Fig. 5a). The explosive 3 is burning, produces gas jet 8 (Fig. 5a), and creates jet rocket thrust 7 which is moving, accelerating, pistons 4 in direction to the center of sphere 1 .

It is very important to simultaneously ignite all fuses 2 of explosive 3. In only this case, the explosive begin to move at the same time into target mass and reach simultaneously its center. They work like rocket engine, accelerate the pistons 4 (having small mass from heavy material) for high speeds in tens of times more that in conventional cumulative explosive. That is the main innovation in the offered method.

The collision of heavy pistons in the center creates a big compression, and the temperature in the fuel capsule initiates the nuclear ignition (Fig. 5b). Nuclear energy heats the explosive gas and increases pressure which a magneto hydrodynamic generator (MHD) converts to electric energy, or the gas (or steam) turbine converts the gas pressure to the mechanical energy (Fig. 6a), or the gas flows to a rocket nozzle and produces a rocket thrust (Fig. 6b).
The cumulative $\mathrm{AB}$ reactor is a cooling unseal method or an injection of water into sphere (Fig. 9b).

The second version is described below. This Version 2 cumulative $A B$ reactor is more suitable for use as a deadly weapon.

The new thermonuclear reactor contains the following (Fig. 7): strong sphere 1, net fuse 2, explosive 3, thin film 4 from heavy material (piston), and fuel pellet 5 into the center of the sphere.

It is very important to simultaneously ignite all the outer surfaces of explosive 3 . In this case only, the explosive begin to move towards mass center and works as rocket engine and accelerate and compress the explosive and film 4 (having small mass) for high speeds in tens of times more that in conventional explosive. That is the main innovation in the offered method.

We can reach the simultaneous ignition of all the outer surfaces of explosive by electric net with small cells. The electric impulse will ignite the entire outer surface of explosive.

The cumulative $\mathrm{AB}$ reactor works in the following way (Figs. 7, 8 and 9). The net fuse 2 (Fig. 8a) simultaneously blows an outer layer of the explosive 3 and an explosive gas

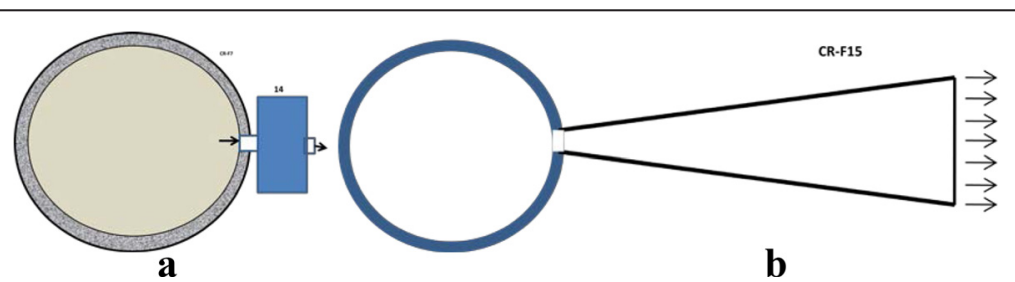

Fig. 6 Final (industrial) work of cumulative AB thermonuclear reactor. Compressed gas from sphere runs to the magneto hydrodynamic (MHD) generator and produces electric energy or runs to gas turbine and produces useful work (a). Notation: 14—conversion of hot gas pressure to electric or mechanical energy, for example, by the magneto hydrodynamic generator (MHD together with condenser), gas (steam) turbine (a) or moving to a rocket nozzle and produces a rocket thrust (b) 


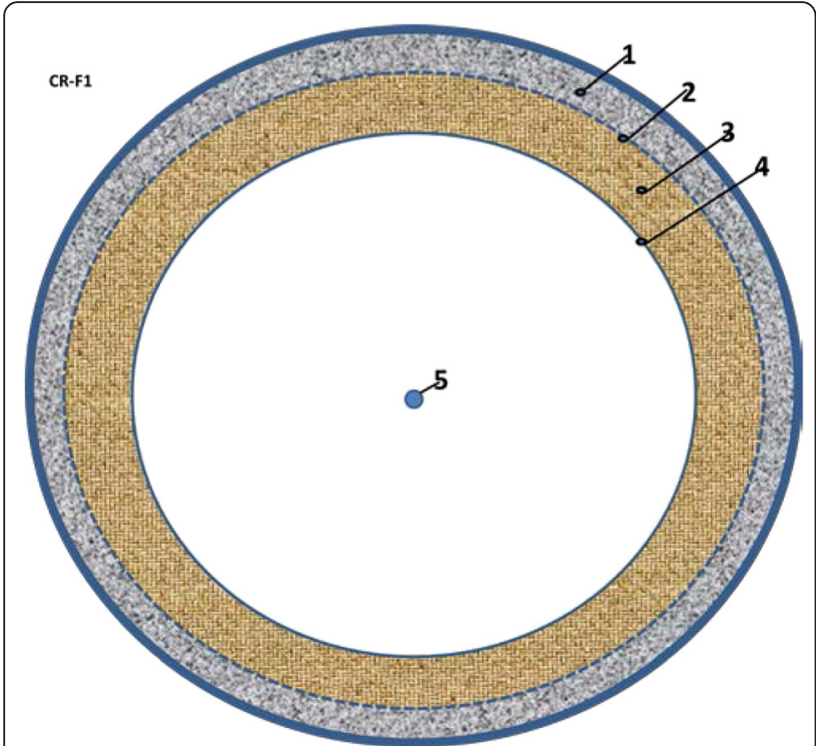

Fig. 7 Impulse cumulative $A B$ thermonuclear reactor (Version 2). Notations: 1-strong sphere, 2-net fuse, 3-explosive, 4-film (piston) of heavy material, and 5-nuclear fuel

6 is pushing the explosive 3 to the center of the sphere. The outer surface of explosive 3 is burning 8 (Fig. $8 \mathrm{~b}$ ) and create jet rocket thrust 7 which is moving, accelerating, and compressing the explosive and thin film 4 in a direction to the center 5 of the sphere 1 .

As a result, the film (piston) made from heavy material which bumps with a high speed (about $30 \mathrm{~km} /$ s) and produced a high pressure (million atmospheres). This pressure is acting with more time than laser pressure and reaches to the center of the fuel capsule (Fig. 9a). The thermonuclear fuel capsule explodes (Fig. 9b). The heavy material brakes the explosion and increases the time and efficiency of the thermonuclear reaction. If installation is used as a reactor, after MHD or turbine, the cooling liquid (for example, water) is injected into the strong sphere. It is converted into hot gas (steam) and rotates the turbine blades (Fig. 6a).

If Version 2 is used as a reactor, when gas is finished, the top semi-sphere opens and embeds a new explosive and the process is repeated. We may have 2-3 explosive spheres for a continuous work.

\section{Advantages of the suggested method compared to the laser method}

The offered reactor and method have the following advantages in comparison with the conventional laser reactor:

1. Cumulative $A B$ reactor is cheaper by thousands of time because it does not have gigantic and very expensive laser installations (see Fig. 1).

2. It is more efficient because the laser efficiency converts only $1-1.5 \%$ electric energy into the light beam. In suggested $A B$ reactor, all the underused (for compression) explosive energy remains in the spherical tank and utilized in MGD or turbine. AB reactor cannot have coefficient $Q$ significantly less 1 . Moreover, it has heat efficiency more than the conventional heat engines because it has very high compression ratio. It can use conventional very high power engine in military transportation.

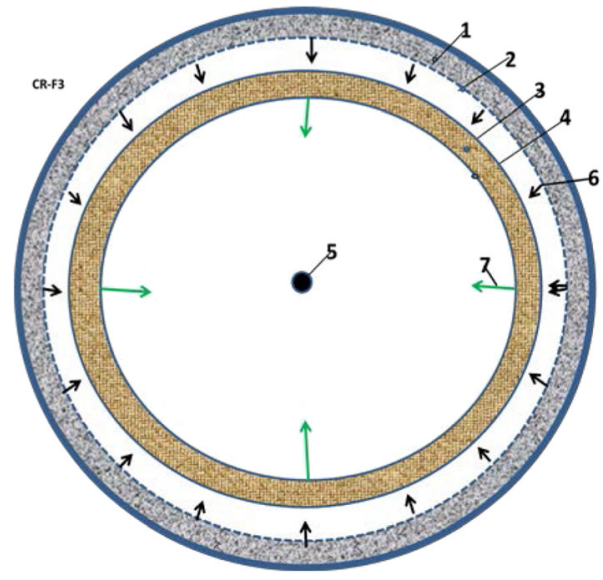

a

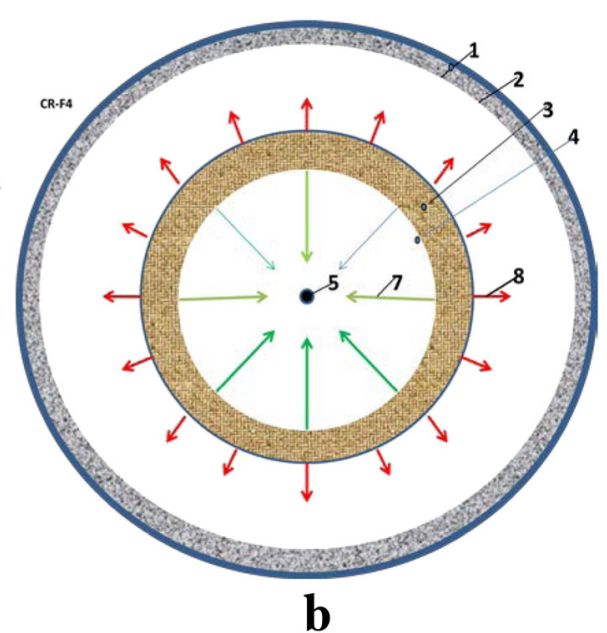

Fig. 8 Work of cumulative $A B$ thermonuclear reactor (Version 2). Notations: (a) initial layer explosive, (b) rocket part of explosive. 1-5 are the same with those in Fig. 7, 6 is pressure of initial layer of explosion, 7 is moving of explosive (force from jetted gas rocket thrust), 8 is flow of reactive explosive gas 


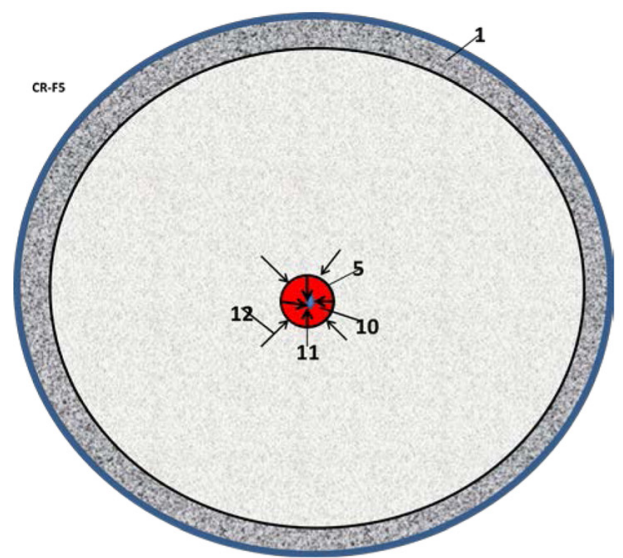

$\boldsymbol{a}$

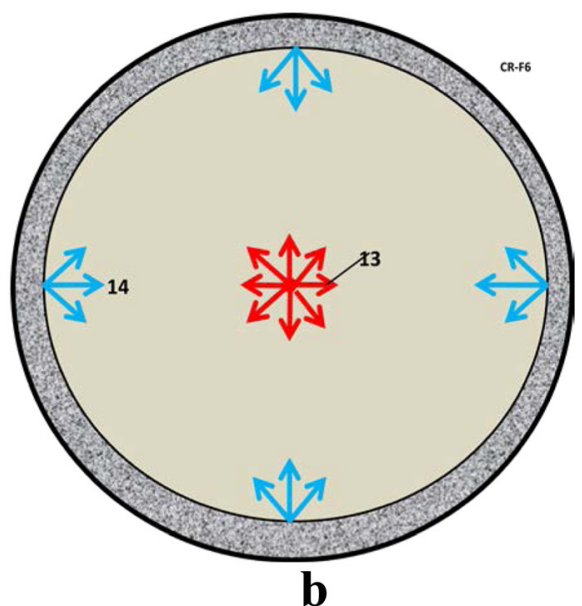

Fig. 9 Work of cumulative AB thermonuclear reactor (Version 2). Notations: (a) final of a fuel compression, (b) thermonuclear explosion and injection of work and cooling liquid (for example, water). 10 is heavy piston (one is in final presses), blocks fuel and increases time for nuclear reaction; 11-brake pressure; 12-outer pressure (shock wave from explosive); 13-thermonuclear exposure of fuel; 14-injection of a work and cooling liquid (for example, water after MHD)

3. The offered very important innovation (accelerating of the explosive by the rocket thrust) allows increasing the top speed of the piston mass 4 from the conventional sound (shock wave) speed $3-\mathrm{km} / \mathrm{s}$ up about $30 \mathrm{~km} / \mathrm{s}$. Only this innovation increases the thermonuclear ignition criterion in 100 times in comparison with the conventional cumulative explosion (see computation). This makes this method available for thermonuclear reaction.

4. Cumulative $A B$ reactor gives compression of the fuel capsule much more than the current laser installations.

5. This compression has a longer time (up to $10^{-3}-10^{-5} \mathrm{~s}$ ) than a light beam pressing (impulse $10^{-9}-10^{-12} \mathrm{~s}$ ), because heavy mass 4 (piston) has many times (10-30) more mass of a capsule (pellet, micro- balloon) 5 . This pressure is supported by rocket gas and shock wave coming from moving explosive gas. This pressure reaches the center of the capsule with a high speed of heavy mass 4 (not sound speed as in laser pressure) increasing the temperature and compressing, with probability of thermonuclear reaction in the fuel capsule.

6. The heavy mass 4 (piston) (having high nuclear numbers $\mathrm{A}$ and $\mathrm{Z}$ ) does not allow the nuclear particles easily to fly apart. That increases the reaction time and reactor efficiency.

7. The suggested $A B$ thermonuclear reactor is small (diameter about $1-1.3 \mathrm{~m}$ or less up $0.3 \mathrm{~m}$ ), is light (mass about $1.7 \mathrm{t}$ or less up $150 \mathrm{~kg}$ for Version 2), and may be used in the transport vehicles and aviation.

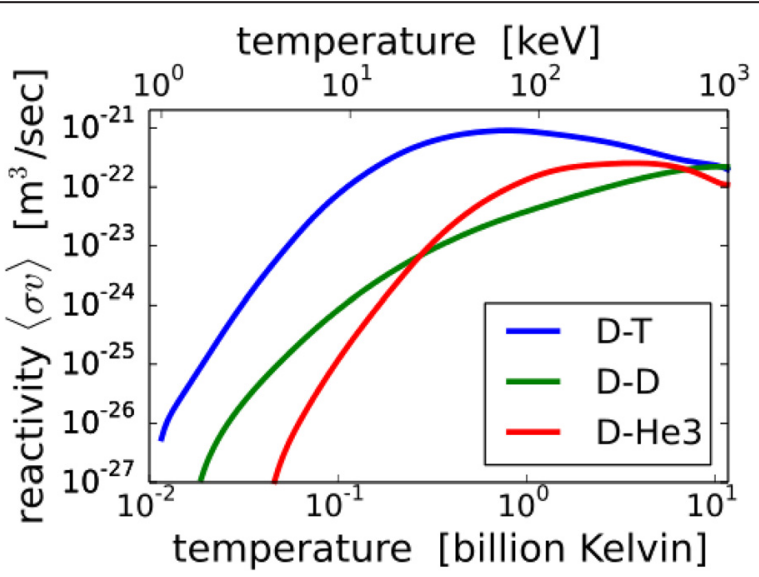

Fig. 10 Reactivity is requested for thermonuclear reaction 
8. The water may protect the material of the sphere from neutrons.

9. It is possible (see computations) that the efficiency of $A B$ reactor will be enough for using as fuel only the deuterium which is cheaper then tritium in thousands times ( $1 \mathrm{~g}$ tritium cost about 30,000 US dollars).

10. Pullet with high-compressed gas fuel may be used instead the frozen/liquid cold $\mathrm{D}+\mathrm{T}$ fuel. That is more compatible for practice.

\section{Results}

Theory, computation, and estimation of cumulative $A B$ reactor and comparison with current ICF

\section{Estimation of laser method (ICF)}

For comparison of the laser and the offered cumulative $A B$ methods, we estimate the current laser method.

Typical laser installation for ICF has the power $5 \mathrm{MJ}$ and deliver to pellet about 20-50 kJ energy. The pullet has the 1-10 mg liquid (frozen) fuel $\mathrm{D}+\mathrm{T}$ (density $200 \mathrm{~kg} / \mathrm{m}^{3}$ ), the diameter of the fuel pullet is about 1-2 $\mathrm{mm}$, and the diameter of an evaporative coating is $4-10 \mathrm{~mm}$.

Let us take the delivered energy $E=50 \mathrm{~kJ}$, the volume of the coating $v=5 \mathrm{~mm}^{3}$, and the specific weight of coating $\gamma=400 \mathrm{~kg} / \mathrm{m}^{3}$ (molar weight $\mu=10$ ).

For these data and instant delivery of laser energy, the maximum pressure in cover is

$$
p=\frac{E}{v}=\frac{5 \times 10^{4}}{5 \times 10^{-9}}=10^{13} \frac{N}{\mathrm{~m}^{2}}=10^{8} \mathrm{~atm}
$$

But we do not know what part this pressure transfer to the fuel pellet.

The number of nuclear in $1 \mathrm{~m}^{3}$ of covering is

$$
\begin{aligned}
n & =\frac{\gamma}{\mu m_{p}}=\frac{0.4 \times 10^{3}}{10 \times 1.67 \times 10^{-27}} \\
& =2.4 \times 10^{28}\left[\mathrm{~m}^{-3}\right]
\end{aligned}
$$

Here, $m p=1.67 \times 10^{-27}$ is the mass of nucleon (proton) [kg].

The temperature of the evaporating cover is

$$
T=\frac{p}{n k}=\frac{10^{13}}{2.4 \times 10^{28} 1.38 \times 10^{-23}}=3 \cdot 10^{7} \quad[\mathrm{~K}]
$$

Here, $k=1.38 \times 10^{-23}$ Boltzmann constant, $/ \mathrm{K}$. The speed of evaporated covering is

$$
\begin{aligned}
V & =\left(\frac{8 k T}{\pi \mu m_{p}}\right)^{0,5} \\
& =\left(\frac{8 \times 1.38 \times 10^{-23} 3 \times 10^{7}}{3.14 \times 10 \times 1.67 \times 10^{-27}}\right)^{0.5} \\
& =2.51 .10^{5} \mathrm{~m} / \mathrm{s}=251 \mathrm{~km} / \mathrm{s}
\end{aligned}
$$

The time of evaporating for the thickness of the covering $l=2 \times 10^{-3} \mathrm{~m}$ is

$$
t=\frac{l}{V}=\frac{2 \times 10^{-3}}{2.51 \times 10^{5}}=8.10^{-9} \mathrm{~s}
$$

Let us to consider now the process into pellet.

The density of fuel particles is

$$
\begin{aligned}
n_{f} & =\frac{\gamma}{\mu m_{p}}=\frac{200}{2.5 \times 1.67 \times 10^{-27}} \\
& =4.8 .10^{28} \frac{1}{\mathrm{~m}^{3}}
\end{aligned}
$$

where $\mu=2.5$ is average molar mass of fuel $\mathrm{D}+\mathrm{T}$.

The frozen (liquid) fuel, after converting in gas, has a temperature of about $T=4 \mathrm{~K}$.

The pressure average speed $V_{\mathrm{n}}$ of the particles after conversion of the fuel into gas (plasma) and sound speed $V_{f}$ to fuel gas at temperature $4 \mathrm{~K}$ are

$$
\begin{aligned}
p_{f} & =n_{f} k T=4.8 \times 10^{28} \times 1.38 \times 10^{-23} \times 4 \\
& =2.65 \times 10^{6} \mathrm{~N} / \mathrm{m}^{2}=26.5 \mathrm{~atm}, V_{n} \\
& =\left(\frac{8 k T}{\pi \mu m_{p}}\right)^{1 / 2}=\left(\frac{8 \times 1.38 \times 10^{-23} \cdot 4}{3.14 \times 2.5 \times 1.67 \times 10^{-27}}\right)^{1 / 2} \\
& =183 \frac{\mathrm{m}}{\mathrm{s}}, V_{f}=\left(\frac{p_{f}}{\rho_{f}}\right)^{1 / 2}==\left(\frac{2.65 \times 10^{6}}{200}\right)^{1 / 2} \\
& =115 \mathrm{~m} / \mathrm{s} .
\end{aligned}
$$

Additional fuel pressure in center of the pellet from two opposing sound wave bump-up is

$$
\begin{aligned}
p_{s} & =\rho_{f}\left(2 V_{f}\right)^{2} / 2=200 \times(2 \times 115)^{2} / 2 \\
& =5.3 \times 10^{6} \quad \mathrm{~N} / \mathrm{m}^{2}=53 \mathrm{~atm}
\end{aligned}
$$

Fuel temperature in the center of the small mass pellet where two opposing sound (shock) wave bump-up happen is

$$
\begin{aligned}
T & =\frac{\pi \mu m_{p}\left(V_{n}+V_{f}\right)^{2}}{8 k} \\
& =\frac{3.14 \times 2.5 \times 1.67 \times 10^{-27}(183+115)^{2}}{8 \times 1.38 \times 10^{-23}}
\end{aligned}
$$

In reality, the full pressure and temperature in the center of the capsule are much more. We compute ONLY 
the sound wave. Any shock wave is faster at short distance than the sound wave. But in our case, this computation is very complex.

The current inertial reactors have the maximal rate of fuel compressing in the center of pellet about

$$
\xi \approx 600
$$

Criterion of ignition (for radius of pullet $R_{\mathrm{o}}=0.02 \mathrm{sm}$ and solid or liquid fuel $\left.\rho_{\mathrm{o}}=0.2 \mathrm{~g} / \mathrm{cm}^{3}\right)$ is

$$
\rho R=\rho_{o} R_{o} \xi^{2 / 3}=0.2 \times 0.02 \times(600)^{2 / 3}=0.28<1
$$

where $\rho$ is in $\mathrm{g} / \mathrm{cm}^{3}$ and $R$ is in $\mathrm{cm}$. That value is not useful enough $(0.28<1)$.

You can imagine-with just a small effort, we will fulfill the criterion of ignition! Focus your attention in the very low temperature of the fuel (9). For this temperature, the criterion may be wrong or the area of the ignition located into the center of the pullet may be very small that energy is very few for ignition of all fuel.

\section{Estimation of cumulative $A B$ reactors}

The proposed cumulative $A B$ reactor is an internal rocket engine which accelerates the small piston from a heavy material by cumulative explosion (Figs. 4, 5, 6, and 7). This piston bumps into the pellet of contained nuclear fuel and compresses and heats the pellet up to very high values, producing a nuclear reaction. The most important innovation is the design, the cumulative explosion which works as rocket engine and produces a final speed of the small piston 15 (and more, from $2 \mathrm{~km} / \mathrm{s}$ to $30 \mathrm{~km} / \mathrm{s}$ ) times and piston energy $(15)^{2}=225$ times more than a convention explosion. Below is not a project but the estimations of the typical parameters of $\mathrm{AB}$ reactors.

\section{Final speed of the piston}

Let us estimate the offered design. It is well known that the final speed $V$ of rocket is

$$
V=-W_{\mathrm{e}} \ln \frac{M_{\mathrm{k}}}{M}=-W_{\mathrm{e}} \ln \mu_{\mathrm{k}}
$$

where $W_{\mathrm{e}}$ is the speed exhaust gas of the rocket, $\mathrm{m} / \mathrm{s}$; $M_{k}$ is the final mass of the rocket, $\mathrm{kg} ; M$ is the initial mass of the rocket, $\mathrm{kg} ; \mu_{k}=M_{k} / \mathrm{M}$ is the ratio of the final and initial mass of the rocket.

The distance $L$ (acceleration way) of the rocket is

$$
\begin{aligned}
& L=g_{0}^{-1} W_{\mathrm{e}}^{2} \nu_{0}\left[1-\mu_{\mathrm{k}}\left(1-\ln \mu_{\mathrm{k}}\right)\right] \quad \text { if } \\
& \mu_{\mathrm{k}}<0.05 \text { then } L \approx g_{0}^{-1} W_{\mathrm{e}}^{2} v_{0}
\end{aligned}
$$

In (12)-(13), the notations used are the following: $g_{o}=9.81 \mathrm{~m} / \mathrm{s}^{2} \sim 10 \mathrm{~m} / \mathrm{s}^{2}$ is Earth acceleration and $v_{\mathrm{o}}=M g_{o} /$ $P_{\mathrm{o}}$ is an initial thrust-to-weight, $N / N$.
The rocket engine uses the solid fuel $W_{\mathrm{e}}=2400$ $2800 \mathrm{~m} / \mathrm{s}$, liquid fuel $W_{e}=3000-3400 \mathrm{~m} / \mathrm{s}$, and hydrogen-oxygen up $W_{e}=4000 \mathrm{~m} / \mathrm{s}$.

The explosive matters have the following:

TNT: specific energy $E_{\mathrm{s}}=4.184 \mathrm{MJ} / \mathrm{kg} \approx 4.2 \mathrm{MJ} / \mathrm{kg}$, density $\rho=1650 \mathrm{~kg} / \mathrm{m}^{3}$, speed of detonation $6900 \mathrm{~m} / \mathrm{s}$ Dynamite: specific energy up $E_{\mathrm{s}}=7 \mathrm{MJ} / \mathrm{kg}$, standard $s=5.3 \mathrm{MJ} / \mathrm{kg}$, density $\rho=1400 \mathrm{~kg} / \mathrm{m}^{3}$, speed of detonation $6000 \mathrm{~m} / \mathrm{s}$.

From $E=m V^{2} / 2$, we get the average speed of exhaust gas for TNT:

$$
\begin{aligned}
W_{\mathrm{e}} & =\left(2 E_{\mathrm{s}}\right)^{1 / 2}=\left(2 \times 4.184 \times 10^{6}\right)^{1 / 2} \\
& =2893 \mathrm{~m} / \mathrm{s}
\end{aligned}
$$

Maximum pressure of explosive is

$$
\begin{aligned}
p & =\frac{E}{v}=\frac{E m}{m v}=E_{\mathrm{s}} \rho=4.184 \times 10^{6} \times 1650 \\
& =6.9 .10^{9} \frac{N}{\mathrm{~m}^{2}}=6.9 .10^{4} \mathrm{~atm}
\end{aligned}
$$

where $v$ is the volume of the explosive, $\mathrm{m}^{3} ; E_{\mathrm{s}}=E / m$ is the specific energy of explosion, $\mathrm{J} / \mathrm{kg}$.

The density of particles and temperature of TNT explosion at the initial moment of explosion (exhaust gas has average $\mu=20$ ):

$$
\begin{aligned}
n & =\frac{\rho}{\mu m_{p}}=\frac{1650}{20 \times 1.67 \times 10^{-27}} \\
& =4.94 \times 10^{28} \mathrm{~m}^{-3}, \quad T=\frac{p}{n k} \\
& =\frac{6.9 \times 10^{9}}{4.94 \times 10^{28} 1.38 \times 10^{-23}}=10.1 \times 10^{3} \quad \mathrm{~K}
\end{aligned}
$$

Let us estimate the final speed of the piston for data: mass of explosive is $M=2 \mathrm{~kg}$, mass of the piston $M_{\mathrm{k}}=20 \mathrm{mg}=2 \times 10^{-5} \mathrm{~kg}, W_{\mathrm{e}}=2650 \mathrm{~m} / \mathrm{s}$.

$$
\begin{aligned}
V & =-W_{\mathrm{e}} \ln \frac{M_{\mathrm{k}}}{M}=-2650 \ln \frac{2 \times 10^{-5}}{2} \\
& =2650 \cdot 5 \cdot 2.3 \approx 30475 \mathrm{~m} / \mathrm{s} \approx 30 \mathrm{~km} / \mathrm{s}
\end{aligned}
$$

Let us find the minimal acceleration distance $L$ of the piston (minimal distance from the lower part of the explosive to the center of the sphere). For Version 1, we receive:

$$
\begin{aligned}
& \text { From } L=g_{0}^{-1} W_{\mathrm{e}}^{2} v_{o}, \quad v_{o}=M g_{0} / F, m_{\mathrm{c}}=\rho V_{\mathrm{d}} S, \\
& \qquad \begin{array}{l}
F=m a=m_{\mathrm{c}} W_{\mathrm{e}}=\rho V_{\mathrm{d}} S W_{\mathrm{e}}, \quad v_{o}=M g_{0} / \rho V_{\mathrm{d}} S W_{\mathrm{e}}, \\
\text { we get } \quad L=\frac{M W_{\mathrm{e}}}{\rho V_{\mathrm{d}} S}=\frac{2 \times 2.6 \times 10^{3}}{1.65 \times 10^{3} \times 30 \times 0.2}=0.46 \quad \mathrm{~m}
\end{array}
\end{aligned}
$$

where $g_{\mathrm{o}}=9.81 \mathrm{~m} / \mathrm{s}^{2}$ is the gravitation; $W_{\mathrm{e}}$ is the 
speed of rocket exhaust gas, $\mathrm{m} / \mathrm{s} ; \rho$ is the rocket fuel density, $\mathrm{kg} / \mathrm{m}^{3} ; V_{\mathrm{d}}$ is the rate (speed) of combustion of the rocket fuel, $\mathrm{m} / \mathrm{s} ; S$ is the initial area of the combustion that created rocket thrust, $\mathrm{m}^{2} ; F$ is the initial rocket thrust, $\mathrm{N} / \mathrm{m}^{2}, M$ is the mass of the rocket fuel, $\mathrm{kg}$. We can change speed $V_{\mathrm{d}}$ to add special additives.

\section{Temperature $T$ and pressure $p$ in pellet}

After compressing, the piston is (for piston speed $V=3 \cdot 10^{4} \mathrm{~m} / \mathrm{s}$ and density of piston $\rho=2 \cdot 10^{4} \mathrm{~kg} / \mathrm{m}^{3}$, $\mu=200)$ :

$$
\begin{aligned}
p & =\frac{\rho(2 V)^{2}}{2}=\frac{2 \cdot 10^{4}\left(2 \cdot 3 \cdot 10^{4}\right)^{2}}{2} \\
& =3.6 \cdot 10^{13} \quad \frac{\mathrm{N}}{\mathrm{m}^{2}}=3.6 \cdot 10^{8} \quad \mathrm{~atm}
\end{aligned}
$$

We take here piston speed $2 \mathrm{~V}$ because we have two opposed pistons.

Temperature

$$
\text { From } \begin{aligned}
E & =\frac{\mu m_{p} V^{2}}{2}=\frac{3 k T}{2} \text { we have } T \\
& =\frac{\mu m_{p} V^{2}}{3 k}
\end{aligned}
$$

The mixture $\mathrm{D}+\mathrm{T}$ has $\mu=2.5$, the piston about $\mu=200$ (for example: tungsten has $\rho=19.34 \times 10^{3} \mathrm{~kg} / \mathrm{m}^{3}$, $\mu=184$; uran-238 has $\rho=19.1 \times 10^{3} \mathrm{~kg} / \mathrm{m}^{3}, \mu=238$; and lead has $\rho=11.35 \times 10^{3} \mathrm{~kg} / \mathrm{m}^{3}, \mu=207$ ).

The temperature of mixture $\mathrm{D}+\mathrm{T}$ is

$$
\begin{aligned}
& \text { For fuel } \mathrm{D}+\mathrm{T} T=\frac{\mu m_{p} V^{2}}{3 k} \\
&= \frac{2.5 \times 1.67 \times 10^{-27}\left(3 \times 10^{4}\right)^{2}}{3 \times 1.38 \times 10^{-23}} \\
&= 91 \times 10^{3} \quad K=7.84 \mathrm{eV}, \\
& \text { For piston } \quad T=\frac{\mu m_{p} V^{2}}{3 k} \\
&=\frac{200 \times 1.67 \times 10^{-27}\left(3 \times 10^{4}\right)^{2}}{3 \times 1.38 \times 10^{-23}} \\
&=7.26 \times 10^{6} \quad \mathrm{~K}=626 \mathrm{eV},
\end{aligned}
$$

The mass of the piston is $10-30$ times more than the mass of the fuel, and the piston has direct contact to the fuel. That means that the fuel has a temperature about seven million degrees. That is less the needed value $10 \mathrm{keV}$ but in thousands of time, it is more than the needed value in a laser method. In the offered method, we have also very more pressure that in the laser method. The high pressure significantly decreases the needed temperature because it decreases the needed distance between nuclear particles.

\section{Estimation of the criterion of ignition in the cumulative $A B$} reactor

The process of compression converted the solid-liquid fuel into gas. According to (7), the initial pressure this gas has is $p_{o}=26.5 \mathrm{~atm}$. In accordance with (19), the final pressure is about $p=3.6 \cdot 10^{8} \mathrm{~atm}$. The rate of fuel compression is

$$
\xi=\frac{p}{p_{0}}=\frac{3.6 \times 10^{8}}{26.5} \approx 1.37 \times 10^{7}
$$

(Comparing this reached value with the maximum $\xi=600$ in the laser method, the cumulative $A B$ reactor has compression many times more than the laser method).

That means that the liner size of fuel pellet will be $(\xi)^{1 / 3}=240$ times less. If the capsule has an initial diameter $D=0.1 \mathrm{~cm}$ (fuel mass $=21 \cdot 10^{-6} \mathrm{mg}, \rho_{o}=$ $0.2 \mathrm{~g} / \mathrm{cm}^{3}$ ), it has $R=0.05 / 240=2.08 \times 10^{-4} \mathrm{~cm}$. The offered cumulative $\mathrm{AB}$ thermonuclear reactor produced direct compression almost a thousands times greater than the usual shock wave laser compression machines at the center of a fuel pullet. The density of the fuel will be $\rho=\rho_{o} \xi=0.2 \times 1.4 \times 10^{7}=2.8 \times$ $10^{6} \mathrm{~g} / \mathrm{cm}^{3}$.

The criterion of the inertial ignition is

$$
\rho R=2.8 \times 10^{6} \times 0.208 \times 10^{-3}=585>1
$$

It is half a thousand times MORE than the needed $(585>1>0.28)$. That means we can use the deuterium which is a thousand times cheaper instead of the very expensive tritium. The corresponding reactions are

$$
\begin{aligned}
& D+T \rightarrow{ }^{4} \mathrm{He}(3.5 \mathrm{MeV})+n(14.1 \mathrm{MeV}) \\
& D+D \rightarrow T(1.01 \mathrm{MeV})+p(3.02 \mathrm{MeV}) 50 \% \\
& D+D \rightarrow{ }^{3} \mathrm{He}(0.82 \mathrm{MeV})+n(2.45 \mathrm{MeV}) 50 \%
\end{aligned}
$$

The deuterium cannot be used in the laser reactor because it requests in 100 times more ignition criterion then $D+T$. But, as you see in (23), it may be used in the $\mathrm{AB}$ reactor (Fig. 10).

The ${ }^{3} \mathrm{He}$ received in the deuterium reaction may be used in the next reactions:

$$
\begin{gathered}
D+{ }^{3} \mathrm{He} \rightarrow{ }^{4} \mathrm{He}((3.6 \mathrm{MeV})+p(14.7 \mathrm{MeV}) ; \\
{ }^{3} \mathrm{He}+{ }^{3} \mathrm{He} \rightarrow{ }^{4} \mathrm{He}+2 p(12.9 \mathrm{MeV}) .
\end{gathered}
$$

They produce only high-energy protons which can be directly converted in electric energy. The last reactions do not produce radio isotopic matters (no neutrons).

Reaction $\mathrm{D}+\mathrm{D}$ has the other distinct advantages: 
1. It produces the protons which energy can be converted directly to electric energy.

2. It produces tritium which is expensive and may be used for thermonuclear reaction.

3. It produces less and low-energy neutrons which create radioactive matters.

The other important advantage is using the pellets with compression gas fuel. Let us take a microballoon having fuel gas with $p_{\mathrm{o}}=100$ atm, radius $0.1 \mathrm{~cm}$, and temperature $300 \mathrm{~K}$. The mass fuel will be $4.19 \mathrm{mg}$.

The compression rate is $\xi=p / p_{o}=3.6 \times 10^{8} / 100=$ $3.6 \times 10^{6}$. The liner size decreases by $(\xi)^{1 / 3}=153$ times. The radius of compressed fuel pellet will be $R=0.1 / 153=0.65 \times 10^{-3} \mathrm{~cm}$. The initial density is

$$
\begin{aligned}
\rho_{0} & =\frac{\mu m_{p} p_{0}}{k T_{0}}=\frac{2.5 \times 1.67 \times 10^{-27} 10^{7}}{1.38 \times 10^{-23} 300} \\
& =10 \frac{\mathrm{kg}}{\mathrm{m}^{3}}=10^{-2} \frac{\mathrm{g}}{\mathrm{cm}^{3}}
\end{aligned}
$$

and the inertial criterion is

$$
\rho R=\rho_{0} R_{0} \xi^{2 / 3}=10^{-2} 0.1 \times\left(3.6 \times 10^{6}\right)^{2 / 3}=23.5>1 .
$$

The criterion is good for compressed fuel $\mathrm{D}+\mathrm{T}$, but it is small for fuel $\mathrm{D}+\mathrm{D}$. For fuel $\mathrm{D}+\mathrm{D}$, we must decrease pressure in pellet up $400 \mathrm{~atm}$ or increase diameter (and power) our installation.

Compressed micro-balloon is more comfortable for laboratory working because it is unnecessary to store fuel at a lower temperature.

\section{Estimation of other parameters of the cumulative $A B$ reactor \\ Thermonuclear energy}

One milligram $\left(10^{-6} \mathrm{~kg}\right)$ of thermonuclear fuel $\mathrm{D}+\mathrm{T}$ has energy.

Number of nucleus:

$$
n_{1}=\frac{M}{\mu m_{p}}=\frac{10^{-6}}{2.5 \times 1.67 \times 10^{-27}}=2.4 \times 10^{20}
$$

One pair of nuclear $\mathrm{D}+\mathrm{T}$ produces energy $E_{1}=$ 17.6 MeV. The $n_{1}$ nuclear particles contain the energy

$$
\begin{aligned}
E & =0.5 n_{1} E_{1}=0.5 \times 2.4 \times 10^{20} 17.6 \times 10^{6} \\
& =21.1 \times 10^{26} \mathrm{eV}=21.1 \times 10^{26} 1.6 \times 10^{-19} \\
& =3.38 \times 10^{8} \mathrm{~J}
\end{aligned}
$$

If coefficient efficiency of the cumulative $A B$ reactor is $\eta=0.3,1 \mathrm{mg}$ of fuel produces the energy 100 million joules. If we make one explosion per second, the installation has the power of 100 million watts. The part of this energy will be produced inside the fuel micro-capsule fuel pellet $\left(3.5 \mathrm{MeV}\right.$ from $\left.{ }^{4} \mathrm{He}, E=6.72 \times 10^{7} \mathrm{~J}\right)$, and most of the energy (14.1 MeV from neutrons) will be produced into the big containment sphere.

Conventional coefficient of nuclear reactor efficiency is about 0.3 , and the steam (gas) turbine is about 0.9.

\section{Energy is delivered by piston to fuel capsule is $E=m V^{2} / 2$}

For $m=30 \mathrm{mg}$, the piston speed is $V=3 \times 10^{4} \mathrm{~m} / \mathrm{s}$ and the final piston energy is $E=13.5 \times 10^{3} \mathrm{~J}$. That is less than the typical energy 20-50 kJ delivered by laser installation. But laser energy is spent in vaporizing the cover of the fuel pellet, and only a small part as shock wave reaches the center of fuel pellet mass. In the cumulative $A B$ reactor, all piston energy passes directly into the target fuel pellet. The piston energy is easy to increase up to $100 \mathrm{~kJ}$ by increasing the piston mass and piston speed (also by using more explosive). The piston mass hinders the fuel micro-balloon and increases the nuclear reaction time many times.

Part of this energy will be used for the ionization of the fuel. One milligram of fuel, for its ionization, requests $E=n_{1} \times 13.6 \mathrm{eV}=522 \mathrm{~J}$, compression of solid fuel about $E=624 \mathrm{~J}$, and compression of gas fuel from $p=$ $100 \mathrm{~atm} E=105 \mathrm{~J}$. That is a small part of the derived piston energy.

\section{Pressure and temperature in sphere after chemical explosive}

Let us take the volume of sphere as $1 \mathrm{~m}^{3}$ and the mass of explosive TNT $2 \mathrm{~kg}$. The energy from chemical explosive is $E_{\mathrm{ch}}=m E_{s}=2 \times 4.2 \times 10^{6}=8.4 \times 10^{6} \mathrm{~J}$. Here, $E_{\mathrm{s}}=4.2 \times 10^{6} \mathrm{~J} / \mathrm{kg}$ is the specific energy of TNT explosive $\mathrm{J} / \mathrm{kg}$. The final pressure of TNT gas after acceleration of piston is

$$
p=E_{\mathrm{ch}} / v=8.4 \times 10^{6} / 1=8.4 \times 10^{6} \mathrm{~N} / \mathrm{m}^{2}=84 \mathrm{~atm} .
$$

The temperature is

$$
\begin{aligned}
T & =\frac{p}{n_{0} k}, \quad \text { where } n_{0}=\frac{M}{\mu m_{p}} \\
& =\frac{2}{20 \times 1.67 \times 10^{-27}}=5 \times 10^{25} \frac{1}{m^{3}}, \\
T & =\frac{8.4 \times 10^{6}}{5 \times 10^{25} 1.38 \times 10^{-23}}=10 \times 10^{3} \quad \mathrm{~K},
\end{aligned}
$$

Here, $\mu=20$ for exhaust gas of TNT. The reaction of TNT is $2 \mathrm{C}_{7} \mathrm{H}_{5} \mathrm{~N}_{3} \mathrm{O}_{6}=3 \mathrm{~N}_{2}+5 \mathrm{H}_{2} \mathrm{O}+7 \mathrm{CO}+7 \mathrm{C}$. 


\section{Estimation of pressure and temperature after nuclear explosion}

Let us find the pressure and temperature after thermonuclear explosive the one mg fuel $\mathrm{D}+\mathrm{T}$.

The number of nuclear particles in sphere $1 \mathrm{~m}^{3}$ is

$$
n_{n}=\frac{M}{\mu m_{p}}=\frac{10^{-6}}{2.5 \times 1.67 \times 10^{-27}}=2.4 \times 10^{20} \frac{1}{\mathrm{~m}^{3}}
$$

The full thermonuclear energy is

$$
\begin{aligned}
E_{\mathrm{n}} & =0.5 n_{n} E_{1}=0.5 \times 2.4 \times 10^{20} 17.6 \times 10^{6} \\
& =21.1 \times 10^{26} \mathrm{eV}=3.38 \times 10^{8} \mathrm{~J}
\end{aligned}
$$

If the coefficient efficiency of the thermonuclear reaction is $\eta=0.3$ in volume $1 \mathrm{~m} 3$ :

$$
\begin{aligned}
p & =\frac{\eta\left(E_{\mathrm{n}}+E_{\mathrm{ch}}\right)}{v}=\frac{0.3\left(3.38 \times 10^{8}+8.4 \times 10^{6}\right)}{1} \\
& =1.038 \times 10^{8} \approx 10^{8} \frac{\mathrm{N}}{\mathrm{m}^{2}}=1000 \mathrm{~atm}
\end{aligned}
$$

The total pressure-nuclear explosive together with chemical explosive-is $p \approx 1000$ atm.

The temperature of gas mixture of explosive plus nuclear fuel is

$$
\begin{aligned}
T & =\frac{p}{\left(n_{0}+n_{n}\right) k}=\frac{10^{8}}{\left(2.6 \times 10^{25}+2.4 \times 10^{20}\right) \times 1.38 \times 10^{-23}} \\
& =279 \times 10^{3} \mathrm{~K}
\end{aligned}
$$

As you can see, the temperature is high, but that is impulse engine. This temperature may be active in just a very short time. Between explosions, the installation is cooling from non-use.

\section{Thickness of sphere cover}

Assume the sphere is made from conventional steel having safety tensile stress $\sigma=50 \mathrm{~kg} / \mathrm{mm}^{2}=5 \times 10^{8} \mathrm{~N} / \mathrm{m}^{2}$. The full tensile force is $F=\pi r^{2} p=3.14 \times 0.5^{2} \times 10^{8}=$ $0.785 \times 10^{8} \mathrm{~N}$. The requested area of steel is $S_{r}=F / \sigma=$ $0.785 \times 10^{8} / 5 \times 10^{8}=0.157 \mathrm{~m}^{2}$. The thickness of sphere wall is $\delta=S_{r} / 2 \pi r=0.157 / 2 \times 3.14 \times 0.5=0.05 \mathrm{~m}$. The mass of sphere is $M_{c} \approx \gamma S_{S} \delta=7800 \times 4.536 \times 0.05=$ $1769 \mathrm{~kg}$. Here, $S_{s}$ is the average surface of the sphere.

If we use the more strong material for sphere wall, for example: $1 \mu \mathrm{m}$ iron whisker having safety tensile stress $\sigma \approx 400 \mathrm{~kg} / \mathrm{mm}^{2}=4 \times 10^{9} \mathrm{~N} / \mathrm{m}^{2}$, we decrease the sphere's mass by four to eight times. We can also make the sphere wall from composite materials (example, an artificial fiber carbon or glass having safety stress $\sigma \approx 100-150 \mathrm{~kg} / \mathrm{mm}^{2}$ and density $\gamma=$ $\left.1500-2700 \mathrm{~kg} / \mathrm{m}^{3}\right)$.

\section{Cooling the sphere by water}

If explosions are very frequent, we then can decrease the wall or/and gas temperature by injection of the chilled or room-temperature water. The water also protects our installation from high-energy protons, in other words, it behaves as a shielding material.

Let us estimate the amount of water which decreases the temperature and pressure of gas (at most steam $\mathrm{H}_{2} \mathrm{O}$ ) into the sphere for magnitudes acceptable for current steam turbines: $T=400{ }^{\circ} \mathrm{C}=672 \mathrm{~K}$. The critical point of water (triple point) is $T=273{ }^{\circ} \mathrm{C}$, $p=22 \mathrm{MPa}$.

Heating $1 \mathrm{~kg}$ water from 20 to $100{ }^{\circ} \mathrm{C}$ requests energy $E=C_{p} \Delta T=4.19 \times 80=333 \mathrm{~kJ}$, evaporation $-r=$ $2260 \mathrm{~kJ}$, and heating of steam up $400{ }^{\circ} \mathrm{C}-E=C_{p} \Delta T=$ $1.05 \times 300=315 \mathrm{~kJ}$. The total amount of water heat energy is $E_{\mathrm{w}}=333+2260+315=2908 \mathrm{~kJ} / \mathrm{kg}$. The total mass of water for nuclear efficiency $\eta=1$ equals $M_{\mathrm{w}}=E$ / $E_{\mathrm{w}}=3.4 \times 10^{8} / 2.9 \times 10^{6}=117 \mathrm{~kg}$. For $\eta=0.3, M_{\mathrm{w}}=35 \mathrm{~kg}$. The $2-3 \mathrm{~cm}$ of water thickness protects the installation from high energy of neutrons produced by reaction $\mathrm{D}+\mathrm{T}$.

Unfortunately, the injection of water before decompressing strongly decreases the efficiency of installation.

\section{Run protons and heavy nuclear particles}

The physic directory by Kikoin, Moscow, 1975, p. 953 gives the following equation for running the protons and charged heavy particles inside gas at pressure $1 \mathrm{~atm}$

$$
R_{\mathrm{x}}(E)=\frac{m_{\mathrm{x}}}{m_{\mathrm{p}}} R_{\mathrm{p}}\left(\frac{m_{\mathrm{p}}}{m_{\mathrm{x}}} E\right)
$$

Where $R_{\mathrm{x}}$ is the run of the investigated particles, $m_{\mathrm{x}}$ is the mass of the investigated particles, $m_{\mathrm{p}}$ is the mass of proton, $R_{\mathrm{p}}$ is the run of the known particles in a known environment, and $E$ is the energy of particles in $\mathrm{MeV}$. The run of proton in $\mathrm{H} 2$ at pressure $1 \mathrm{~atm}$ is in the Table 1.

For particles ${ }^{4} \mathrm{He}(3.5 \mathrm{MeV})$ in reaction $\mathrm{D}+\mathrm{T}$ under the piston pressure $p=108 \mathrm{~atm}$, the run is

$$
\begin{aligned}
R_{\mathrm{x}}(E) & =\frac{m_{\mathrm{x}}}{m_{\mathrm{p}}} R_{\mathrm{p}}\left(\frac{m_{\mathrm{p}}}{m_{\mathrm{x}}} E\right) / p \approx \frac{4}{1} R_{\mathrm{p}}\left(\frac{1}{4} 1 \times 3.5\right) / 10^{8} \approx 4 \times 10 / 10^{8} \\
& =4 \times 10^{-7} \mathrm{~cm} \approx 4 \times 10^{-6} \mathrm{~mm}
\end{aligned}
$$

The closed run has a proton.

That means that all the energy of the charges particles after nuclear reaction is used for heating other "cold"

Table 1 Run (range) of proton in gas $\mathrm{H} 2$ at pressure $1 \mathrm{~atm}$

\begin{tabular}{llll}
\hline Energy $E[\mathrm{MeV}]$ & 1 & 10 & 100 \\
Run $R[\mathrm{~cm}]$ & 10 & 5.102 & 2.104 \\
\hline
\end{tabular}


particles. If probability of an initial reaction is more than $10 \mathrm{keV} / 3500 \mathrm{keV}=1 / 350$, the chain reaction and ignition will occur.

In the cumulative $\mathrm{AB}$ reactor, these conditions are in whole fuel capsule, and in the laser reactor, many times, lower conditions may be only in the center of the fuel capsule (collision of the imposed shock waves). If reacted particles run out the center of the capsule, its energy will be wasted.

The runway of neutrons is large and has very complex function of energy and conditions. Environment.

\section{Converting the nuclear energy of cumulative $A B$ reactor to electric, mechanical energy or a rocket thrust}

The best means for converting a cumulative $\mathrm{AB}$ reactor nuclear energy is the MHD generator which converts with high efficiency the high temperature and high-pressure plasma directly in electric energy. Together with capacitors, it can produce continuous electric currency. Short impulse work of reactor allows to cool the reactor by impulse injection of the cooler (or conventional cooling) and protect the cumulative $A B$ reactor installation from a very high temperature.

The second way for converting a cumulative $A B$ reactor nuclear energy is conventional heat exchanger and gas turbine, as the cooler may use the FLiBe-melted mix of fluoride salts of lithium and beryllium.

The third way is injection of water inside sphere and steam turbine as description over.

\section{Using the cumulative $A B$ reactor as an impulse space rocket engine}

There are good prospects (possibility) to use the suggested cumulative $\mathrm{AB}$ reactor as an impulse rocket engine.

If plasma will flow from the sphere to the space, the average speed $V$ of the jet is

$$
\text { From } \begin{aligned}
E & =\frac{m V^{2}}{2} \quad \text { we get } V=\left(\frac{2 E}{m}\right)^{1 / 2} \\
& =\left(\frac{2 \times 10^{8}}{2}\right)^{1 / 2}=10^{4} \frac{\mathrm{m}}{\mathrm{s}}
\end{aligned}
$$

Here, $E$ is the nuclear energy in one impulse $1 \mathrm{mg}$ nuclear fuel, J, and $m$ is the mass injected to the outer space (together with conventional explosive), $\mathrm{kg}$.

The received speed $V=10 \mathrm{~km} / \mathrm{s}$ is three times more than the current exhaust chemical speed (it may be up to $25 \mathrm{~km} / \mathrm{s}$ ). If a space apparatus has a mass of $1 \mathrm{t}$, the ship speed changes in $V 2=(\mathrm{m} 1 / \mathrm{m} 2) V 1=20 \mathrm{~m} / \mathrm{s}$ in one strong impulse.
More importantly, the next possibility is of the rocket powered by the cumulative $\mathrm{AB}$ reactor. Any matter from any planets, asteroids, and space body may be used as fuel used for increasing the derivation of impulses. For example, assume that the captured solid object moving through space is is composed of some water, and we filled rocket tanks using that mined planet, comet, or asteroid water. From (35) and the Law of equal impulse, we have from every impulse

$$
V_{1}=\left(2 E m_{1}\right)^{1 / 2} / m_{2}=\left(2 \times 10^{8} \times 16\right)^{1 / 2} / 10^{3}=56.6 \mathrm{~m} / \mathrm{s}
$$

Here, $V_{1}$ is the added speed $m 1$ mass jet $\mathrm{kg}, m_{1}=$ $14 \mathrm{~kg}$ of water $+2 \mathrm{~kg}$ of explosive, and $m_{2}$ is the mass of the space apparatus.

We take the frequency of shots $1 \mathrm{~Hz}$, but current automatic guns have tens to hundreds of shots per second.

\section{Discussion}

About 60 years ago, scientists conducted Research and Development of a thermonuclear reactor that promised then a true revolution in the energy industry and, especially, in humankind's aerospace activities. Using such reactor, aircraft could undertake flights of very long distance and for extended periods and that, of course, decreases a significant cost of aerial transportation, allowing the saving of ever-more expensive imported oilbased fuels. (As of mid-2006, the US DoD has a program to make aircraft fuel from domestic natural gas sources). The temperature and pressure required for any particular fuel to fuse is known as the Lawson criterion $L$. The Lawson criterion relates to plasma production temperature, plasma density, and time. The thermonuclear reaction is realized when $L$ is a more certain magnitude. There are two main methods of nuclear fusion: inertial confinement fusion (ICF) and magnetic confinement fusion (MCF).

Existing thermonuclear reactors are very complex, expensive, large, and heavy. They cost many billions of US dollars and require many years for their design, construction, and prototype testing. They cannot stably achieve the nuclear ignition and the Lawson criterion. In the future, they will have a lot of difficulties with acceptable cost of nuclear energy, with converting the nuclear energy to conventional energy, and with small thermonuclear installation suitable for transportation or space exploration. Scientists promise an industrial application of thermonuclear energy after 10-15 years additional researches and new billions of US dollars in the future. But old methods will not allow to reach it in nearest future.

In inertial confinement, many scientists thought that short pressure $\left(10^{-9}-10^{-12} \mathrm{~s}\right)$, which they can reach by laser 
beam, compress the fuel capsule, but this short pressure only creates the shock wave which produced the not large pressure and temperature in a limited range area in center of fuel capsule. The scientists try to reach it by increasing NIF, but plasma from the initial vaporization of the cover of the fuel capsule does not allow to deliver big energy. After laser beam, the fuel capsule is a "naked" capsule. A capsule cannot keep the high-energy particles of the nuclear ignition and loss them. Producing the power laser beam is very expensive and has very low efficiency (1-1.5\%).

The offered method does not have these disadvantages. It directly presses fully the fuel capsule to high pressure and temperature by piston, it covers the capsule by piston mass in 10-30 times more than the mass of fuel, and it protects the fuel by the heavy elements having high number of nucleons $\mathrm{A}$ and charges $\mathrm{Z}$. They reflect the light protons, $\mathrm{D}$, and $\mathrm{T}$, repel high-energy reacted particles $\left({ }^{4} \mathrm{He}, \mathrm{p}\right)$ back to fuel, and significantly (in hundreds time) increase the conformation time.

It is important also that all chemical (explosive) energy not used for compression of the fuel capsule remain into the sphere and may be used for useful work.

The cumulative idea cannot be used for thermonuclear reaction in its classical form. Produced pressure and temperature are not enough for thermonuclear reaction. The main author innovation is using the rocket thrust of explosive for acceleration piston for a very high speed (from 2 up to $30 \mathrm{~km} / \mathrm{s}$ and more), that increases the kinetic energy of the piston 225 times.

The author noted that the mass of the fuel and the piston is very small and allows reaching the high rocket speed of pressing by piston.

The method possibly allows using reaction $\mathrm{D}+\mathrm{D}$ (instead $\mathrm{D}+\mathrm{T}$ ) with cheap nuclear fuel $\mathrm{D}$ (tritium is very expensive-about 20,000 USD for $1 \mathrm{~g}$ ). It also allows using the compressed fuel gas at room temperature.

The method also may be used for getting the metal hydrogen, which has super-conductivity at room temperature and high thermal capacity.

\section{Conclusions}

The author offers a new, small, cheap, and cumulative inertial thermonuclear reactor, which increases the pressure and temperature of a nuclear fuel in thousands times, reaches the ignition and full thermonuclear reaction. The cumulative $\mathrm{AB}$ reactor, herein offered by its originator, contains several innovations.

The main of them is using a moved explosive, which allows to accelerate the special rocket piston to a very high speed (more than $30 \mathrm{~km} / \mathrm{s}$ ) which (as it is shown by computations) compresses the fuel capsule in million times and heating up million degrees of temperature. The cumulative reactor allows to get the Lawson criterion in hundreds of times higher than necessary $(\rho R=585>1$, Eq. (23)).

The offered reactor is small and cheap and may be used for cheap electricity, as engine for Earth transportation (train, truck, sea-going ships, aircraft), for space apparatus, and for producing small and cheap and powerful weapons. Closed author ideas are in [1-4], the last researches of the conventional nuclear reactors ICF, MCF are in [5-11], and used nuclear data is in [12].

\section{Competing interests}

The author declares that he has no competing interests.

\section{Author's information}

Short biography of Bolonkin, Alexander Alexandrovich (1933-): Alexander A. Bolonkin was born in the former USSR. He holds a doctoral degree in aviation engineering from Moscow Aviation Institute and a post-doctoral degree in aerospace engineering from Leningrad Polytechnic University. He has held the positions of senior engineer in the Antonov Aircraft Design Company and Chairman of the Reliability Department in the Clushko Rocket Design Company. He has also lectured at the Moscow Aviation Universities. Following his arrival in the USA in 1988, he lectured at the New Jersey Institute of Technology and worked as a Senior Scientist at NASA and the US Air Force Research Laboratories.

Bolonkin is the author of more than 250 scientific articles and books and has 17 inventions to his credit. His most notable books include The Development of Soviet Rocket Engines (Delphic Ass., Inc., Washington, 1991); Non-Rocket Space Launch and Flight (Elsevier, 2006); New Concepts, Ideas, Innovation in Aerospace, Technology and Human Life (USA, NOVA, 2007); Macro-Projects: Environment and Technology (NOVA, 2008); Human Immortality and Electronic Civilization, 3rd Edition, (Lulu, 2007; Publish America, 2010): Life and Science. Lambert Academic Publishing, Germany, 2011, 205 pgs. ISBN: 978-3-84730839-3. http://www.archive.org/details/Life.Science.Future.biographyNotes ResearchesAndlnnovations; Ultra-Cold Thermonuclear Synthesis: Criterion of Cold Fusion. USA, 2015. http://viXra.org/abs/1507.0158; Femtotechnologies and Revolutionary Projects. Lambert, USA, 2011. 538 p. 16 Mb. ISBN:978-3-8473-0839-0. http://viXra.org/abs/1309.0191,

Home page: http://Bolonkin.narod.ru.

\section{Acknowledgements}

The author wishes to acknowledge R.B. Cathcart and J.J. Friedlander for correcting the author's English and offering useful advices and suggestions.

Received: 28 October 2015 Accepted: 27 February 2016

Published online: 29 March 2016

\section{References}

1. Bolonkin AA (2011) Femtotechnologies and revolutionary projects. Lambert, USA, 2011. 538 p. 16 Mb. ISBN:978-3-8473-0839-0. http://viXra.org/abs/1309.0191

2. Bolonkin AA (2014) Innovations and new technologies (v2). Lulu, USA, 2014. 465 pgs. 10.5 Mb, ISBN: 978-1-312-62280-7. https://archive.org/details/ Book5InnovationsAndNewTechnologiesv2102014/

3. Bolonkin AA (2015) Cumulative thermonuclear AB-reactor. Vixra, USA. http://viXra.org/abs/1507.0053

4. Bolonkin AA (2009) Converting of any matter to nuclear energy by ABgenerator. Am J Eng Appl Sci 2(4):683-693, http://viXra.org/abs/1309.0200

5. Chen $F$ (2011) An indispensable truth: how fusion power can save the planet. Springer, New York. ISBN 978-1441978196

6. Clery D (2013) A piece of the sun. Overlook, New York. ISBN 978-1468304930

7. Dean S (2013) Search for the ultimate energy source: a history of the U.S. fusion energy program. Springer, New York. ISBN 978-1461460367

8. Hoffman, M (2013). "What Is the Lawson criteria, Or how to make fusion power viable". http://www.scienceworldreport.com/articles/5763/20130323/ lawson-criteria-make-fusion-power-viable-iter.htm.

9. Molina A d B (2013) Kinetic simulations of ion transport in fusion devices. Springer, New York. ISBN 978-3319004211 
10. Inertial Confinement Fusion Program Activities, March 2006 Archived May 11, (2009) at the Wayback Machine https://web.archive.org/web/ 20090511180008/http:/www.lle.rochester.edu/pub/progress/MarDOE06.pdf

11. "Initial NIF experiments meet requirements for fusion ignition". Lawrence Livermore National Laboratory. (2010). https://www.Inl.gov/news/initial-nifexperiments-meet-requirements-fusion-ignition\%C2\%A0-new-physicseffectachieves.

12. Kikoin IK (1975) Tables of physical values, Moscow, Atomizdat, (Russian)

\section{Submit your manuscript to a SpringerOpen ${ }^{\odot}$ journal and benefit from:}

- Convenient online submission

- Rigorous peer review

- Immediate publication on acceptance

- Open access: articles freely available online

- High visibility within the field

- Retaining the copyright to your article

Submit your next manuscript at $>$ springeropen.com 